\title{
MTHFR - Ala222Val Effects on Metabolic Syndrome Progression
}

\author{
Katalin Csép*, Eszter Szigeti, Krisztina Szalman
}

University of Medicine and Pharmacy Tirgu Mures, Romania

Objective: Methylene-tetrahydrofolate reductase (MTHFR) is involved in adapting metabolism to environmental challenges by various mechanisms, including the control of gene expression by epigenetic and post-translational changes of transcription factors. Though a metabolic syndrome candidate gene, association studies of its common polymorphism rs1801133 (MTHFR-Ala222Val) remain inconclusive with important ethnic differences, and the effect on disease progression was not addressed. Methods: 307 middle-aged metabolic syndrome patients in a central Romanian hospital setting were investigated metabolically, and genotyped by PCR-RFLP. Disease progression was assessed by the age of onset of metabolic components, as well as development of non-alcoholic fatty liver disease and atherosclerotic complications. Results: The minor allele frequency of rs1801133 was 30.13\%. Metabolic parameters showed no statistically significant differences according to genotype, but variant carriers developed dysglycemia and dyslipidemia earlier $(53.28 \pm 10.8$ vs $59.44 \pm 9.31$ years, $p<0.05$ and $58.57 \pm 11.31$ vs $64.72 \pm 10.6$ years, $p<0.1$ ). While the polymorphism did not influence hepatic complications, an inverse association was found for manifest atherosclerosis ( $\mathrm{OR}=0.49, \mathrm{p}=0.006,95 \% \mathrm{Cl}: 0.29-0.81)$, which may be folate-status dependent, and needs further investigations. Simultaneous analysis with transcription factor polymorphisms (rs1801282, rs8192678) showed that the more protective genotypes were present the later metabolic disturbances developed, and in the presence of the other two variants the apparent protective cardiovascular effect disappeared. Conclusions: The common functional polymorphism rs1801133 may influence metabolic syndrome progression, the age of onset of components and development of atherosclerotic complications. Besides simple additive effects, complex mitigating and aggravating variant interactions may exist, and the protective or predisposing outcome may depend on modifiable environmental factors.

Keywords: metabolic syndrome X, atherosclerosis, non-alcoholic fatty liver disease, methylenetetrahydrofolate reductase, genetic polymorphism

\section{Introduction}

Since the human genome is considered to be relatively unchanged from Stone Age, it could be deduced that the diabesity epidemic may be related to an inadequate interaction between inherited genetic polymorphisms associated with a survival advantage in long starving periods of the past and significantly changed current sedentary lifestyle and food abundance, an explanation formulated by Neel's thrifty hypothesis. Obesity and insulin resistance are considered central disturbances in the development of the much debated clinical entity known as the metabolic syndrome (MetS). Molecules acting as transcription factors, their co-activators and epigenetic regulators as well as posttranslational modifiers, responsible for the direct or indirect control of gene expression at various levels on short and long term, involved in fatty acid oxidation, adipogenesis and lipogenesis play a crucial role in the adaptation of metabolism to environmental challenges, thus their genes are important candidates of disease development.

MTHFR (methylene-tetrahydrofolate reductase) is a key enzyme of folate metabolism, controlling the proportional use of one-carbon units in nucleic acid synthesis and methylation - a major mechanism of epigenetic control and posttranslational changes, as well as methionin synthesis, involving the detoxification of homocysteine associated

* Correspondence to: Katalin Csep

E-mail: genetica.umftgmro@gmail.com with increased oxidative stress. The most common and potentially functional polymorphism rs1801133 (MTHFR Ala222Val) was widely investigated in type 2 diabetes, but less studied, with even more conflicting results in MetS, or non-alcoholic fatty liver disease (NAFLD) and atherosclerotic cardiovascular disease (CVD) associated with it.[1, 2] While previous studies targeted the assessment of the associated disease risk, the effect on progression was not investigated based on a Pubmed search. Given the controversial findings and lacking data, as well as potential practical implications of a genotype-based case management, we proposed to explore the effect of the polymorphism on the development of MetS components and complications, to our best knowledge uninvestigated in the local population. We hypothesized, that MTHFR polymorphism could influence MetS development by various mechanisms including the epigenetic control at DNA and histone level or by post-translational changes at protein level of transcription factors central for adipogenesis, lipid and glucose metabolism. Preclinical data suggest that adequate co-activator function of PPARGC1A (peroxisome proliferatoractivated receptor- $\gamma$ coactivator $1-\alpha$ ), including its interaction with PPARG2 (peroxisome proliferator-activated receptor- $\gamma$ ), depends on MTHFR activity.[3, 4] Joint effect of the most common polymorphisms of these three master regulators of metabolism by direct and indirect control of gene expression, however, was not previously addressed. 


\section{Methods}

$307 \mathrm{MetS}$ patients in a central Romanian region, diagnosed by the International Diabetes Federation proposed criteria, were recruited from Mureș County Emergency Hospital, and included in the study after obtaining informed written consent, according to the protocol approved by the institutional Ethics Committee.

Disease progression was assessed by the age of onset of metabolic components and development of non-alcoholic fatty liver disease and atherosclerotic complications. NAFLD was assessed as an elevated alanin transaminase level (>30IU/L in males, >19IU/L in females), and/or increased fatty liver index $(>60$, calculated according to the formula developed by Bedogni et al, 2006: e $\mathrm{e}^{0.953^{*} \log _{\mathrm{e}}(\text { triglyc- }}$ erides $)+0.139 * \mathrm{BMI}+0.718^{*} \log _{\mathrm{e}}(\mathrm{GGT})+0.053^{*}$ waist circumference -15.745$) /(1$

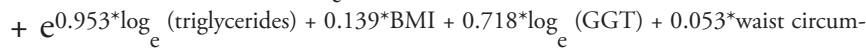
ference -15.745$) \times 100$ ), and/or a positive diagnosis; all cases with liver disorder of known other etiology (i.e. toxic or infectious) were excluded. CVD was considered in clinically manifest atherosclerosis diagnosed as peripheral arteriopathy and/or cerebro- and/or cardiovascular disease.

Genotyping was done by PCR-RFLP using the following primer pairs (Eurogentec) and restriction enzymes (FastDigest, Thermo Scientific):

- 5'-CATCCCTATTGGCAGCTTAC-3'/5'-GACGGTGCGGTGAGAGTG-3', Hinf1 (rs1801133MTHFR/Ala222Val);

- 5'-CAAGTCCTCCAGTCCTCAC-3'/5'GGGGTCTTTGAGAAAATAAGG-3', MspI (rs8192678-PPARGC1A/Gly482Ser);

- 5'-GCCAATTCAAGCCCAGTC-3'/5'-GATATGTTGCAGACAGTGTATCAGTGAAGGAATCGCTTTCCG-3'，Bsh1236I (rs1801282-PPARG2/ Pro12Ala).

Amplification took place after an initial denaturation at $95^{\circ} \mathrm{C}-5 \mathrm{~min}$, in 38 cycles consisting of $95^{\circ} \mathrm{C}-40 \mathrm{sec}$, $57 / 58 / 60^{\circ} \mathrm{C}-40 \mathrm{sec}$ and $72^{\circ} \mathrm{C}-40 \mathrm{sec}$, ended with a final extension $72^{\circ} \mathrm{C}-5 \mathrm{~min}$. Digestion was carried out at $37^{\circ} \mathrm{C}$ in $5 \mathrm{~min}$, followed by electrophoresis in 2\% agarose gels stained with Ethidium Bromide.

Statistical analysis was done with IBM SPSS Statistics 20 , considering results statistically significant if $p<0.05$ (two-tailed). Data are expressed as mean \pm standard deviation (SD), and compared by one-way ANOVA. Categorical variables are expressed as percentage, and examined by Fisher's exact test.

\section{Results}

$307 \mathrm{MetS}$ patients $(61.62 \pm 10.59$ years old; $47.88 \%$ males, $52.11 \%$ females; $57.01 \%$ from urban and $42.99 \%$ from rural area) were investigated as summarized in Table I.

Minor allele frequencies of rs1801133, rs8192678 andrs1801282 in the MetS patients investigated were 30.13, 32.89 and $15.79 \%$, respectively; all polymorphisms were in Hardy-Weinberg equilibrium ( $p>0.05$ ). When calculating with genotype combinations, homo- and heterozygous variant carriers were interpreted together according to the dominant model (rs1801133: Ala/Val+Val/Val; rs8192678: Gly/Ser+Ser/Ser; rs1801282: Pro/Ala+Ala/Ala).

Metabolic parameters according to the possible predisposing genotype combinations are presented in Table II.

To assess genotype dependent MetS development and progression, the age of onset of metabolic disturbances, as well as the risk of NAFLD and CVD was analyzed (Table III and IV).

\section{Discussion}

Progress in genomics calls for a better characterization of genetic variation, confirmation of functional consequences, assessment of gene - gene and environment interactions, testing disease associations in various ethnicities. Possibilities to investigate joint effects of polymorphisms remain limited. Risk scores have little practical utility, especially in older people for adult onset multifactorial disorders be-

Table I. Clinical characterization

\begin{tabular}{lc}
\hline Clinical parameter & Mean \pm SD \\
\hline Body Mass Index-BMI $\left(\mathrm{kg} / \mathrm{m}^{2}\right)$ & $29.9 \pm 4.77$ \\
Males & $30.82 \pm 7.05$ \\
Females & \\
Waist Circumference $(\mathrm{cm})$ & $107.43 \pm 14.25$ \\
Males & $98.11 \pm 14.03$ \\
Females & $125.44 \pm 43.68$ \\
Fasting Glucose $(\mathrm{mg} / \mathrm{dL})$ & $148.12 \pm 21.54$ \\
Systolic Blood Pressure $(\mathrm{mmHg})$ & $87.13 \pm 12.01$ \\
Diastolic Blood Pressure $(\mathrm{mmHg})$ & $203.24 \pm 117.87$ \\
Triglyceride $(\mathrm{mg} / \mathrm{dL})$ & \\
HDL-Cholesterol $(\mathrm{mg} / \mathrm{dL})$ & $48.95 \pm 12.81$ \\
Males & $50.55 \pm 14.95$ \\
Females & $210.7 \pm 54.44$ \\
Total Cholesterol $(\mathrm{mg} / \mathrm{dL})$ & \\
Alanine transaminase $(\mathrm{IU} / \mathrm{L})$ & $30.92 \pm 15.21$ \\
Males & $29.58 \pm 12.61$ \\
Females & $83.67 \pm 17.8$ \\
Fatty Liver Index & \\
\hline
\end{tabular}

Table II. MetS components according to the suspected predisposing genotype combinations*

\begin{tabular}{|c|c|c|c|c|c|}
\hline & $\begin{array}{c}\text { No predisposing } \\
\text { variant }\end{array}$ & rs1801133 & $\begin{array}{c}\text { rs1801133+ } \\
\text { rs8192678 }\end{array}$ & $\begin{array}{c}\text { rs1801133+ } \\
\text { rs1801282 }\end{array}$ & $\begin{array}{c}\text { rs1801133 + rs1801282 } \\
+ \text { rs8192678 }\end{array}$ \\
\hline Body Mass Index (kg/m²) & $28.57 \pm 4.84$ & $33.08 \pm 10.65$ & $31.05 \pm 6.83$ & $30.26 \pm 6.58$ & $29.63 \pm 5.19$ \\
\hline Waist Circumference (cm) & $98.35 \pm 9.81$ & $107.31 \pm 12.59$ & $108.7 \pm 12.61$ & $101.72 \pm 14.92$ & $101.7 \pm 15.78$ \\
\hline Fasting Glucose (mg/dL) & $112.52 \pm 23.6$ & $124.28 \pm 41.3$ & $115.24 \pm 30.88$ & $128.70 \pm 48.2$ & $123.54 \pm 41.65$ \\
\hline Systolic Blood Pressure (mmHg) & $150.1 \pm 20.9$ & $145.48 \pm 21.65$ & $149.5 \pm 18.62$ & $148.59 \pm 19.49$ & $146.07 \pm 22.3$ \\
\hline Diastolic Blood Pressure $(\mathrm{mmHg})$ & $81.8 \pm 7.76$ & $85.79 \pm 12.67$ & $93.49 \pm 12.9$ & $87.83 \pm 12.18$ & $90.32 \pm 11.37$ \\
\hline Triglyceride (mg/dL) & $188.18 \pm 77.9$ & $172.54 \pm 129.65$ & $226.6 \pm 93.5$ & $190.6 \pm 138.4$ & $267.98 \pm 110.15$ \\
\hline HDL-Cholesterol (mg/dL) & $41.14 \pm 6.61$ & $52.47 \pm 18.22$ & $47.29 \pm 7.48$ & $47.06 \pm 20.19$ & $45.8 \pm 15.77$ \\
\hline
\end{tabular}


Table III. Onset of MetS components according to the genotype*

\begin{tabular}{|c|c|c|c|c|c|c|c|}
\hline & & \multicolumn{2}{|c|}{ Type 2 diabetes } & \multicolumn{2}{|c|}{ High Blood Pressure } & \multicolumn{2}{|c|}{ Dyslipidemia } \\
\hline & & Mean \pm SD & $\mathrm{p}$ & Mean \pm SD & $\mathrm{p}$ & Mean \pm SD & $\mathrm{p}$ \\
\hline \multirow{2}{*}{ rs1801133 } & $\mathrm{Ala} / \mathrm{Val}$ or $\mathrm{Val} / \mathrm{Val}$ & $53.28 \pm 10.8$ & \multirow{2}{*}{0.007} & $54.95 \pm 13.57$ & \multirow{2}{*}{0.32} & $58.57 \pm 11.31$ & \multirow{2}{*}{0.06} \\
\hline & Ala/Ala & $59.44 \pm 9.31$ & & $52.36 \pm 11.58$ & & $64.72 \pm 10.6$ & \\
\hline \multirow{2}{*}{$\begin{array}{l}\text { rs1801133 + } \\
\text { rs8192678 }\end{array}$} & Ala/Val or Val/Val + Gly/Ser or Ser/Ser & $55.13 \pm 12.02$ & \multirow{2}{*}{0.51} & $54.88 \pm 11.27$ & \multirow{2}{*}{0.79} & $58.38 \pm 13.13$ & \multirow{2}{*}{0.07} \\
\hline & Ala/Ala + Gly/Gly & $56.98 \pm 9.94$ & & $55.68 \pm 9.69$ & & $64.32 \pm 9.7$ & \\
\hline \multirow{2}{*}{$\begin{array}{l}\text { rs1801133+ } \\
\text { rs1801282 }\end{array}$} & $\mathrm{Ala} / \mathrm{Val}$ or $\mathrm{Val} / \mathrm{Val}+$ Pro/Pro & $52.38 \pm 10.25$ & \multirow{2}{*}{0.19} & $53.34 \pm 11.7$ & \multirow{2}{*}{0.69} & $58.48 \pm 10.95$ & \multirow{2}{*}{0.28} \\
\hline & Ala/Ala (MTHFR) + Pro/Ala or Ala/Ala (PPARG2) & $56.73 \pm 8.83$ & & $55.22 \pm 15.65$ & & $62.15 \pm 10.47$ & \\
\hline \multirow{2}{*}{$\begin{array}{l}\text { rs1801133 + } \\
\text { rs8192678 + } \\
\text { rs1801282 }\end{array}$} & Ala/Val or Val/Val + Gly/Ser or Ser/Ser + Pro/Pro & $55.88 \pm 10.93$ & \multirow[b]{2}{*}{0.21} & $57.94 \pm 10.75$ & \multirow[b]{2}{*}{0.38} & $59.05 \pm 12.65$ & \multirow[b]{2}{*}{0.25} \\
\hline & $\begin{array}{l}\text { Ala/Ala (MTHFR) + Gly/Gly + } \\
\text { Pro/Ala or Ala/Ala (PPARG2) }\end{array}$ & $61.60 \pm 7.76$ & & $61.17 \pm 6.24$ & & $64.14 \pm 8.72$ & \\
\hline
\end{tabular}

Table IV. Risk of NAFLD and CVD according to the genotype

\begin{tabular}{|c|c|c|c|c|c|c|c|}
\hline & & \multicolumn{3}{|c|}{ NAFLD } & \multicolumn{3}{|c|}{ CVD } \\
\hline & & OR & $\mathrm{p}$ & $95 \% \mathrm{Cl}$ & OR & $\mathrm{p}$ & $95 \% \mathrm{Cl}$ \\
\hline rs1801133 & Ala/Val+Val/Val vs Ala/Ala & 0.54 & 0.3 & $0.18-1.6$ & 0.49 & 0.006 & $0.29-0.81$ \\
\hline $\begin{array}{l}\text { rs1801133 + } \\
\text { rs8192678 }\end{array}$ & Ala/Val or Val/Val + Gly/Ser or Ser/Ser vs Ala/Ala + Gly/Gly & 0.75 & 0.46 & $0.36-1.55$ & 0.63 & 0.22 & $0.3-1.28$ \\
\hline $\begin{array}{l}\text { rs1801133+ } \\
\text { rs1801282 }\end{array}$ & $\begin{array}{c}\text { Ala/Nal or Val/Val + Pro/Pro vs } \\
\text { Ala/Ala (MTHFR) + Pro/Ala or Ala/Ala (PPARG2) }\end{array}$ & 1.29 & 0.53 & $0.58-2.84$ & 0.57 & 0.25 & $0.25-1.29$ \\
\hline $\begin{array}{l}\text { rs1801133 + } \\
\text { rs8192678 } \\
+ \text { rs1801282 }\end{array}$ & $\begin{array}{c}\text { Ala/Nal or Val/Val + Gly/Ser or Ser/Ser + Pro/Pro vs } \\
\text { Ala/Ala (MTHFR) + Gly/Gly + Pro/Ala or Ala/Ala (PPARG2) }\end{array}$ & 1.5 & 0.45 & $0.57-3.92$ & 0.64 & 0.46 & $0.23-1.79$ \\
\hline
\end{tabular}

cause of lifelong exposure to environmental risk factors. Study of genetic variants influencing disease progression could provide perhaps more valuable information, and by analyses in the same age group and region, long-lasting effects of confounding factors could be better accounted for.

Molecules acting as transcription factors, their co-activators and epigenetic regulators as well as posttranslational modifiers, involved in the direct or indirect control of gene expression, on short and long term, regulating genes involved in metabolism play a crucial role in its adaptation to environmental challenges. The functional polymorphism rs1801133 (C677T; Ala222Val) of MTHFR leads to a thermo-labile form with altered substrate/cofactor affinity and enzyme activity decrease to $1 / 3$ and $2 / 3$ in hetero- and homozygous carriers. [5] Folic acid supplements compensate variant dysfunction, though preventive utility proved in congenital anomalies is uncertain in atherosclerosis. rs1801133 associates with increased homocysteine levels, and was reported inconsistently in association with MetS components and other common disorders (e.g. Alzheimer disease, depression), mainly in Asians and less in Caucasians.[1, 2, 6] Moreover, second-generation antipsychotics related MetS development appears to depend on the polymorphism.[7] Ultraviolet exposure-related lower folate levels may be genotype specific, and could account for ethnic differences.[8] Protective effects reported in cancer, renal failure or NAFLD, may suggest heterozygosis advantage.[9]In the local population, we observed a relatively high variant frequency in both patients and healthy controls, with no significantly increased MetS risk associated.[10] Hyperhomocysteinemia is often considered part of the MetS. Hyperinsulinemia was reported to decrease MTHFR activity, and hyperhomocysteinemia to hinder insulin signaling. [11, 12] We speculated that poly- morphism reduced enzyme activity and insulin resistance in MetS could be mutually aggravating, but accentuated insulin resistance assessed by HOMA and QUICKI indices, though increased in $\mathrm{T}$ allele carriers in both patients and control, did not differ significantly as compared to $\mathrm{CC}$ in our population.[13] As far as MetS disease progression is concerned in patients, variant carriers appear to develop dysglycemia and to a lesser extent dyslipidemia significantly earlier $(53.28 \pm 10.8$ vs $59.44 \pm 9.31$ years, $\mathrm{p}<0.05$ and $58.57 \pm 11.31$ vs $64.72 \pm 10.6$ years, $\mathrm{p}<0.1)$.

The effect on MetS-associated NAFLD and CVD may be indirect or direct, by metabolic changes, inflammation and oxidative stress. Clinical experience suggests that NAFLD is the hepatic manifestation of MetS, resulting from an imbalance between fat supply, formation and consumption, as well as pro- and anti-oxidant action; in fact, triglyceride accumulation in the beginning could be protective by decreasing free fatty acid accumulation and the associated oxidative stress.[14] According to the twohit hypothesis, first steatosis develops, and then hepatitis and fibrosis. While steatosis develops in the majority of obesity cases, progression to hepatitis and fibrosis is rarer, and could have genetic causes. Among the candidate genes, MTHFR was also studied. Hyperhomocysteinemia considered a risk factor for liver disease by increased oxidative stress, activation of pro-inflammatory factors and altered intracellular lipid metabolism, could explain NAFLD association with MTHFR-Val, but was not equivocally demonstrated: it may not exist in Caucasians, and could characterize only subjects homozygous for the variant.[15] In the dominant model we applied, no such effect was seen, similar to neighboring populations [16]. The conflicting results could be related to folate status, as suggested in CVD. rs1801133 is viewed as a proxy for 
hyperhomocysteinemia-a CVD risk factor associated with increased oxidative stress, but genetic association studies are few and inconclusive. $[17,18]$ Our unexpected results may confirm the inverse association between the homozygous genotype for the variant and CVD mortality found in Caucasian populational studies, despite higher homocysteine levels. Possible explanations cited include publication bias, methodological problems and folate status, since lower CVD-mortality may characterize persons with adequate folate intake. Accordingly, geographic and dietary differences might explain the contradictory findings and the significant risk reduction we found in variant carriers despite an earlier onset of metabolic disturbances considered CVD risk factors, but require further investigations, including folate status assessment. Confirmation would emphasizethe importance of genetics and gene-environment interactions in patient management, and may signify the potential inversion of variant effect from predisposing to protective by lifestyle interventions, diet and vitamin supplements. Moreover, the polymorphism was shown to require further tailoring of dietary interventions, i.e. adequate protein intake in case of a hypocaloric diet to prevent sarcopenia in T allele-carriers. [19]

Common functional variants of metabolic master regulators individually or jointly, by independent additive effect within a polygenic system or complex interactions and inter-dependent activity, may influence the MetS phenotype. Lipotoxicity is opposed via adipose tissue remodeling - increased storage capacity by adipocyte hypertrophy and hyperplasia, or via increased use by lipid oxidation. $[20,21]$ According to the adipocentric view, PPARG is an important MetS candidate. As a transcription factor, PPARG2 controls the activity of genes involved in adipocyte development and fat storage, lipid metabolism and inflammation. By expansion of the subcutaneous adipose tissue, however, lipids accumulate selectively in hormonally less sensitive depots, while there is no change in visceral adiposity with direct access to portal circulation.PPARGC1A described initially as a PPARG co-activator, is a multifunctional regulator of various transcription factors. It plays a central role in the regulation of metabolism and energy homeostasis, coordinating metabolic pathways in response to environmental requirements such as food supply, exercise or temperature. It is involved in the control of mitochondrial biogenesis, adaptive thermogenesis, adipocyte differentiation, fatty acid $\beta$-oxidation and glucose metabolism.[21, 22] PPARGC1A and PPARG2 functions are closely related: PPRAGC1A regulates PPARG2, and major metabolic effects of the co-activator are carried out by the nuclear receptor. Relationships of MTHFR with PPARGC1A, PPARG2 and MetS appear manifold, and are little studied. Adequate PPARGC1A activity depends on post-translational changes. A possible mechanism involved in Barker's early origins of adult onset disorders hypothesis could be the imbalanced methylation-acetylation of PPARGC1A dependent on MTHFR.[4] Later in life rs1801133-associated hyperhomocysteinemia causing enhanced PPARGC1A nitrotyrosylation could further impair co-activator function and interaction with PPARG2 [3]. Additionally, PPARG ligation reduces homocysteine levels, and in PPARG2 variant carriers homocysteine levels were found higher,[23] suggesting a bi-directional interaction. Common variants of these metabolic master genes have been rarely studied together, and based on a Pubmed search the combined effect of the three polymorphisms has not been addressed yet, despite interactions suggested by preclinical research and potential practical utility based on limited clinical data in assessing disease susceptibility, prediction of progression or response to interventions. [24] rs 1801282 is a missense mutation (Pro12Ala) in the ligand-independent activation domain of PPARG2, associated with reduced DNA-binding and 30\% decrease in trans-activation.[25] rs8192678 is a missense mutation (Gly482Ser) of PPARGC1A, and though apparently it doesn't alter binding sites for known transcription factors, reduced activity could be explained by impaired interaction efficacy. $[26,27]$ The mutation-containing exon encodes for a domain that appears to interact with PPARG, so a conformational change might reduce binding affinity. Protein structures resulting from the amino-acid sequence changes don't offer clear answers about interactions. In PPARG2, the polymorphism is contained within the variable $\mathrm{A} / \mathrm{B}$ region on the $\mathrm{N}$-terminal end responsible for ligand-independent trans-activation, while the $\mathrm{F}$ region on the C-terminal end appears important for interaction with co-activators. Functional consequences of the polymorphisms on interaction are contradictory. While some found that neither substitution influenced target gene transcription in vitro,[26] others demonstrated that PPARG2 activation by PPARGC1A is compromised in the presence of Ala,[28] confirmed by epidemiologic observations in breast cancer related to alcohol consumption [29]. Petersen et al. suggested an interaction characterized by a Pro allele-specific effect of the PPARG-PPARGC1A complex, but Ruchat et al. reported an independent effect in non-diabetic subjects undergoing glucose tolerance test, higher fasting insulin and HOMA-IR in carriers of both Gly and Ala compared to Ala carrier-Ser/Ser persons, with no difference in Pro/Pro subjects [30].The suggested PPARGC1A co-activator function and interaction with the nuclear receptor PPARG2 dependent on normal MTHFR activity could be impaired in the presence of rs1801133, and presumably the effect could be further modulated in carriers of rs8192678 and rs 1801282 . Though simultaneous presence of polymorphisms did not further aggravate onset of metabolic disturbances, the age of onset of dyslipidemia in case of bothrs 1801133 and rs8192678 remained reduced to the same extent. In the presence of rs 8192678 and rs 1801282, however, the apparent significant protective effect on atherosclerotic complication development of the MTHFR variant disappeared, possibly mitigated by the pro-atherogenic effects of the transcription factor vari- 
ants through metabolic changes, mitochondrial function, oxidative stress and inflammation.

Interpretation of the obtained results has several limitations. In general, lack of accounting for confounding factors may explain the conflicting results in genetic association studies. Assessment of several candidate genes with minor effects if any, additive or in complex interactions, requires investigation on larger samples and still await for the development of adequate study approaches. Stratification according to sex and bodyweight could offer more conclusive results, and insulin, homocysteine and folate status evaluation or the use of more sensitive approaches to assess the hepatic and vascular burden could provide further insight. We analyzed effects according to the widely used dominant model, this approach, however, may be arguable, since there are also reports of over-dominant effects and significant changes observable only in recessive models. Nonetheless, our pathway based approach may represent an alternative to address variant interactions influencing disease progression by simple methodologies. Gene-gene and gene-environment interactions in general, and more importantly in these metabolic master genes need to be addressed, since due to frequency, involvement in common adult-onset disease etiology from the earliest stages of development, and modifiable nature of variant-associated effects by personalized interventions, novel information obtained could have also public health implications.

\section{Conclusions}

By various direct and indirect effects on metabolism, MTHFR may be considered a candidate gene for the MetS. Its common functional polymorphism rs 1801133 may influence metabolic syndrome evolution, the age of onset of components - dysglicemia or dyslipidemia, and development of atherosclerotic complications but not NAFLD, in the local population of Caucasian origin. Simultaneous study with other polymorphisms, such as rs 1801282 and rs8192678 of metabolic master genes possibly influenced by MTHFR suggests that besides simple independent additive effects within a polygenic system responsible for genetic predisposition, complex mitigating and aggravating variant interactions may exist, and the protective or predisposing outcome may depend on modifiable environmental factors.

\section{Conflict of interest}

None to declare.

\section{Acknowledgments}

Molecular genetic analysis was performed on infrastructure provided to Prof. Dr. Bănescu Claudia by grant number 41-042 /2007, CNMP-PNCD II - parteneriate, hereby the authors wish to thank for support.

\section{References}

1. Russo GT, Di Benedetto A, Alessi E, et al. Mild hyperhomocysteinemia and the common C677T polymorphism of methylene tetrahydrofolatereductase gene are not associated with the metabolic syndrome in Type 2 diabetes. J Endocrinol Invest. 2006; 29(3):201-207.

2. Chen AR, Zhang HG, Wang ZP, et al. C-reactive protein, vitamin B12 and C677T polymorphism of N-5,10-methylenetetrahydrofolate reductase gene are related to insulin resistance and risk factors for metabolic syndrome in Chinese population. Clin Invest Med. 2010; 33(5):E290-7.

3. Veeranki S, Givvimani S, Pushpakumar S, Tyagi SC. Hyperhomocysteinemia attenuates angiogenesis through reduction of hif-1alpha and pgc-1alpha levels in muscle fibers during hindlimb ischemia. Am J Physiol Heart Circ Physiol 2014;306:H1116-H1127.

4. Pooya S, Blaise S, Moreno Garcia M, et al. Methyl donor deficiency impairs fatty acid oxidation through PGC- $1 \alpha$ hypomethylation and decreased ER- $\alpha$, ERR- $\alpha$, and HNF- $4 \alpha$ in the rat liver. $J$ Hepatol 2012;57:344-351

5. SNPedia - rs1801133. https://www.snpedia.com/index.php/ Rs1801133

6. Rai V. Methylenetetrahydrofolate Reductase (MTHFR) C677T Polymorphism and Alzheimer Disease Risk: a Meta-Analysis. Mol Neurobiol 2017;54(2):1173-1186.

7. Devlin AM, Ngai YF, Ronsley R, Panagiotopoulos C. Cardiometabolic risk and the MTHFR C677T variant in children treated with secondgeneration antipsychotics. Transl Psychiatry. 2012; 24;2:e71.

8. Cordain L, Hickey MS. Ultraviolet radiation represents an evolutionary selective pressure for the south-to-north gradient of the MTHFR 677TT genotype. Am J Clin Nutr. 2006; 84(5):1243.

9. Trovato FM, Catalano D, Ragusa A, et al. Relationship of MTHFR gene polymorphisms with renal and cardiac disease. World J Nephrol. 2015; 4(1):127-137

10. Csép K, Todoran Butilă A, Bănescu C, Szigeti E. MTHFR - C677T polymorphism and the metabolic syndrome. Acta Medica Marisiensis 2016; 62(Suppl8):103.

11. Uehara SK, Rosa G. Association of homocysteinemia with high concentrations of serum insulin and uric acid in Brazilian subjects with metabolic syndrome genotyped for C677T polymorphism in the methylenetetrahydrofolatereductase gene. Nutr Res. 2008; 28(11):760766.

12. Movva S, Alluri RV, Venkatasubramanian S, Vedicherla B, Vattam KK. Association of methylene tetrahydrofolate reductase C677T genotype with type 2 diabetes mellitus patients with and without renal complications. Genet Test Mol Biomarkers 2011; 15:257-261.

13. Csép K, Todorona Butilă A, Bănescu C - A MHTFR - C6777T génpolimorfizmus hatása az insulin-érzékenységre és a hasnyálmirigy béta-sejt funkcióra. Orvostudományi Értesítő 2017;90(2):10

14. Werstuck GH, Lentz SR, Dayal S. Homocysteine-induced endoplasmic reticulum stress causes dysregulation of the cholesterol and triglyceride biosynthetic pathways. J Clin Invest. 2001; 107:1263-1273.

15. Sun MY, Zhang L, Shi SL, Lin JN. Associations between Methylenetetrahydrofolate Reductase (MTHFR) Polymorphisms and Non-Alcoholic Fatty Liver Disease (NAFLD) Risk: A Meta-Analysis. PLoS ONE 11(4): e0154337.

16. Kasapoglu B, Turkay C, Yalcin KS, Kosar A, Bozkurt A. MTHFR 677C/T and 1298A/C mutations and non-alcoholic fatty liver disease. Clin Med. 2015; 15(3):248.

17. Yang Q, Bailey L, Clarke R, et al.Prospective study of methylenetetrahydrofolate reductase (MTHFR) variant C677T and risk of all-cause and cardiovascular disease mortality among 6000 US adults. Am J Clin Nutr May; 2012; 95(5):1245-1253.

18. Clarke R, Bennett DA, Parish S, et al. Homocysteine and coronary heart disease: meta-analysis of MTHFR case-control studies, avoiding publication bias. PloS Med 9(2):e1001177

19. Di Renzo L, Marsella LT, Sarlo F, et al. C677T gene polymorphism of MTHFR and metabolic syndrome: response to dietary intervention. Journal of Translational Medicine 2014; 12:329.

20. Reddy JK, Hashimoto T - Peroxisomal $\beta$-oxidation and peroxisome proliferator-activated receptor $\gamma$ : An Adaptive Metabolic System. Annu Rev Nutr 2001;21: 193-230.

21. Medina-Gomez G, Gray S, Vidal-Puig A - Adipogenesis and lipotoxicity: role of peroxisome proliferator-activated receptor gamma (PPARgamma) and PPARgammacoactivator-1 (PGC1). Public Health Nutr 2007;10:1132-1137

22. Wu H, Deng X, Shi Y, Su Y, Wei J, Duan H - PGC-1 $\alpha$, glucose metabolism and type 2 diabetes mellitus. Journal of Endocrinology 2016;229:R99R115. doi: 10.1530/JOE-16-0021.

23. Golledge J, Norman PE - Relationship between two sequence 
variations in the gene for peroxisome proliferator-activated receptorgamma and plasma homocysteine concentration. Health in men study. Human Genetics 2008;123(1):35-40

24. Andrulionyte L, Peltola P, Chiasson JL, Laakso M - Single Nucleotide Polymorphisms of PPARD in Combination With the Gly482Ser Substitution of PGC-1A and the Pro12Ala Substitution of PPARG2 Predict the Conversion From Impaired Glucose Tolerance to Type 2 Diabetes The STOP-NIDDM Trial. Diabetes 2006;55(7):2148-2152

25. Deeb SS, Fajas L, Nemoto M, et al - A Pro12Ala substitution in PPARgamma-2 associated with decreased receptor activity, lower body mass index and improved insulin sensitivity. Nature Genet 1998;20:284-287.

26. Nitz I, Ewert A, Klapper M, Doring F - Analysis of PGC-1alpha variants Gly482Ser and Thr612Met concerning their PPARgamma2-coactivation function. Biochemical and Biophysical Research Communications 2007;353:481-486.
27. Choi YS, Hong JM, Lim S, SooKo K, Pak KM - Impaired coactivator activity of the Gly482 variant of peroxisome proliferator-activated receptor c coactivator-1a (PGC-1a) on mitochondrial transcription factor A (Tfam) promoter. Biochemical and Biophysical Research Communications, 2006;344:708-712.

28. Heikkinen S, Auwerx J, Argmann CA - PPARgamma in human and mouse physiology. Biochim. Biophys. Acta 2007;1771:999-1013.

29. Petersen RK, Larsen SB, Jensen DM, et al - PPARgamma-PGC-1alpha activity is determinant of alcohol related breast cancer. Cancer Letters 2012;315:59-68.

30. Ruchat SM, Weisnagel SJ, Vohl MC, Rankinen T, Bouchard C, Pérusse $L$ - Evidence for interaction between PPARG Pro12Ala and PPARGC1A Gly482Ser polymorphisms in determining type 2 diabetes intermediate phenotypes in overweight subjects. Exp Clin Endocrinol Diabetes 2009;117:455-459. 